OnLine Journal of Biological Sciences 9 (4): 86-92, 2009

ISSN 1608-4217

(C) 2009 Science Publications

\title{
The Incidence of Fibromyoma and Polycystic Ovary Syndrome in Women in Trinidad (2000-2003)
}

\author{
E.O. Uche-Nwachi, A. Odekunle, M. Welch, D. Bowleg, B. Cardron, \\ K. Gaebolae, T. Humes, K. Modise and L. Tlale \\ Anatomy Unit, Faculty of Medical Sciences, \\ University of the West Indies, St. Augustine, Trinidad and Tobago, West Indies
}

\begin{abstract}
Problem statement: Before this study was done there were no reports of similar studies in Trinidad whereas similar study had been reported from other parts of the World including the Caribbean sub-region. The present study was thus designed to investigate the incidence and demographics of leiomyomata and polycystic ovary syndrome in women in two health institutions in Trinidad during the period from 2000-2003, inclusive. The study also sought to establish the prevalence of the established risk factors viz. age, ethnicity, parity and gravidity and to examine their relationship with the two disease conditions. Approach: The study population included only women who were diagnosed with or treated for these conditions at the Mt. Hope Medical Science Complex and San Fernando General Hospital in Trinidad and Tobago during the specified period. Results: The most common presenting complaints were vaginal bleeding and colicky lower abdominal pain for fibroids and polycystic ovarian syndrome respectively. The majority of the fibroid cases were aged between 40 and 49 years. Similarly most of the patients with polycystic ovary were aged between 20 and 29 years. Hysterectomy was the most frequent surgical intervention. Prior to this, patients were placed on haematinics for anaemia and analgesics for pain. Conclusion: The present study has provided the database that could be harnessed in improving the health care delivery system for the population of Trinidad in different ways as discussed in the text of the article.
\end{abstract}

Key words: Fibroid, polycystic ovary, myomectomy, hysterectomy

\section{INTRODUCTION}

Leiomyomata commonly known as flbroid, is a common type of benign tumor that grows slowly within the wall of the uterus ${ }^{[1,2]}$. They are made up of smooth muscle and fibrous tissues and their dimensions can vary widely, from pea-sized to the size of a full-term pregnancy. They may also vary in number, ranging from one to many ${ }^{[3]}$. Polycystic ovary syndrome is a very common endocrinopathy in women that is often associated with multiple, small, fluid-filled cysts in the ovaries ${ }^{[4]}$. Leiomyomata and polycystic ovary syndrome can cause a variety of problems for women of childbearing age. There are different types of fibroids depending on their size and location in the uterine wall. Fibroids may occur singly or in groups in different parts of the uterine wall. Small fibroids do not cause problems, but larger ones may affect menstruation or cause lower abdominal pain, urinary or bowel problems, complications during pregnancy, anaemia ${ }^{[3]}$ and infertility ${ }^{[4]}$. Polycystic ovarian syndrome is an endocrinopathy in women characterized by excessive androgen production leading to irregular ovulation which may result in infertility. It is sometimes also referred to as "hyperandrogen anovulation syndrome" or "Stein Leventhal Syndrome",[5]. In this condition, both ovaries become enlarged with multiple, fluid-filled cysts $^{[6]}$. The exact cause of fibroids is uncertain, but risk factors such as diet ${ }^{[7]}$, obesity, stress, heredity and high concentrations of estrogen appear to play a significant role in their development ${ }^{[8]}$. Since both of these conditions are most often asymptomatic, women who do not know that they have Leiomyomata and or polycystic ovary syndrome will be driven to seek medical help only when a secondary problem occurs, as a result of either one, or both of these conditions.

Although Leiomyomata and polycystic ovary syndrome can cause irreparable damage to the female reproductive organs in some cases it may only lead to abnormal menstruation, pelvic discomfort and infertility ${ }^{[9]}$, not a great deal of research has been done

Corresponding Author: A. Odekunle, Anatomy Unit, Faculty of Medical Sciences, University of the West Indies, St. Augustine, Trinidad and Tobago Tel: 1-868-7702096 
in the Caribbean and more specifically in Trinidad and Tobago on the incidence of these conditions

The motivation for this study came from the realization that there was enough pertinent data to do a retrospective study on the incidence of these conditions in these health institutions. Furthermore, in the belief that women should be more aware of the signs and predisposing factors of polycystic ovary syndrome and uterine leiomyomata therefore, rationalization of a retrospective study is apparent. It is also our conviction that the limitations of such a study are far surpassed by the benefits that will be derived from the study.

Our methodology consists of a retrospective study focusing on post-pubertal and pre-menopausal women diagnosed with either leiomyomata or polycystic ovary during the 4 year period from 2000-2003. It this study, we propose to calculate the incidence, discover the presenting complaints and investigate the management prescribed for leiomyomata and polycystic ovary syndrome. We will also note any existing correlation between these conditions, age and ethnicity.

\section{MATERIALS AND METHODS}

Study design: This was a retrospective, descriptive and hospital based study, designed to assess the incidence of Leiomyomata and polycystic ovarian syndrome in women in two principal health institutions in Trinidad. The chosen design was felt to be most suitable for the study as it provided valuable information with regards to the distribution of leiomyomata and polycystic ovarian syndrome in these institutions. In addition, the design also allowed for the study to be performed relatively quickly and inexpensively, while providing a foundation for further study.

Target population: The target population studied included female patients of child-bearing age in Trinidad that had been reported as presenting with polycystic ovarian syndrome and leiomyomata at the Women's Maternity Hospital of The Eric Williams Medical Sciences Complex (Mt. Hope) and San Fernando General Hospital. The method of simple random sampling for each year, throughout the 4-year period ranging from 2000-2003 was utilized. We reviewed records on files at the aforementioned hospitals for this time period to obtain specific criteria such as ethnicity, parity and other personal information required for the study. Furthermore, admissions records were also used to determine the incidence for the specified period.

Inclusion criteria: The study included women of childbearing age in Trinidad that reported and presented with leiomyomata and polycystic ovarian syndrome at The Eric Williams Medical Sciences Complex (Women's Maternity Hospital, Mt. Hope) and San Fernando General Hospital.

Exclusion criteria: Excluded from the study were prepubertal and postmenopausal females.

Demographics: Our study consisted of a descriptive, retrospective study in which we obtained Medical Records from the Eric Williams Medical Sciences Complex (Women's Maternity Hospital Mt Hope) and San Fernando General Hospital taking into consideration age, ethnicity, parity and gravidity.

Description of outcome measurements: The study sought to determine the incidence of leiomyomata and polycystic ovarian syndrome in Trinidad and to distinguish amongst the various types of leiomyomata. Another goal of the study was to determine if there was any possible correlation between fibromyoma and polycystic ovarian syndrome with age, ethnicity, parity and gravidity. The data collected was analyzed and presented in the forms of graphs and various types of charts, showing fluctuation in the incidence of the conditions over the years, as well as indicating which ethnicity and age range were affected based on our findings.

The Ethics Committee of the Faculty of Medical Sciences, The University of the West Indies (St. Augustine Campus) approved the protocol of the study and the necessary ethical precautions were implemented. There was a special coding system developed and employed for confidentiality and identity protection, while simultaneously allowing for efficient data collection. In addition, all data entry and other computer manipulation of the attained findings were performed on a secure, password-protected computer again as a precautionary measure. The study design allowed for relatively simple statistical manipulation, however initially it appeared to be problematic. There was a great deal of research done on fibromyoma and polycystic ovarian syndrome in Europe and the United States, however, using estimations based on the findings in the previously mentioned researches would not be relevant to our study. Due to the lack of research done on this subject in the region it was advised and indeed necessary to locate an estimated value to represent the proportion of fibroid cases that would be relevant to the study being performed here in Trinidad. A pilot study was carried out using data obtained from Eric Williams Medical Science Complex, Women's Maternity Hospital, Mt. Hope in the year 1999 to 
generate the necessary p-value (0.35) necessary for further statistical manipulation. There was a confidence level of $95 \%$ with a standard error of $5 \%$, making the chances of obtaining results using these values quite high. Using the value obtained from the pilot study, with additional statistical values obtained they were then substituted into a "dichotomous" formula that was use to find the minimum sample size for fibromyoma and polycystic ovarian syndrome respectively. It was calculated that 450 cases (350-fibromyoma and 100polycystic ovarian syndrome) should provide enough statistical power to detect the differences deemed important including potential confounding variables. In actuality, 600 cases were reviewed per year, allowing for greater accuracy and reducing error. Once all the data had been obtained, analysis was carried out using the Statistical Package for Social Scientists (SPSS) program and these findings are expressed in results.

\section{RESULTS}

The total number of case obtained for the study was 2400. Of this number, $473(20 \%)$ were fibroid cases, $51(2 \%)$ were polycystic ovarian syndrome cases and $42(2 \%)$ were women who had both fibroids and polycystic ovarian syndrome. This data was obtained from Mt. Hope Maternity Hospital and San Fernando General Hospital for the period 2000-2003, inclusive. The $\mathrm{P}$ values for this data were 0.35 and 0.01 for fibroids and polycystic ovary respectively, with a confidence interval of $95 \%$.

Figure 1 shows the frequencies of fibroids and polycystic ovaries and the occurrences of both in Trinidad from 2000 to 2003. In 2000 there were 116 (19\%) cases of fibroids and $10(2 \%)$ for polycystic ovarian syndrome. In 2001 there were 144 (24\%) cases for fibroids, while for polycystic ovarian syndrome there were $15(3 \%)$ cases. In 2002 there were 92 (15\%) cases of fibroids and $10(2 \%)$ polycystic ovary cases. In 2003 there 121 (20\%) fibroids cases while there were $17(3 \%)$ cases of polycystic ovarian syndrome Fig. 2 shows percentage parity and gravidity in Trinidadian women with either fibroids or Polycystic Ovarian syndrome and with both (2000-2003). Figure 2 also shows that on average there were many more cases of fibroids than polycystic ovary syndrome. There were $405(86 \%)$ cases of gravidity and $255(54 \%)$ cases of parity for fibroids out of the total 473 cases. Polycystic ovary syndrome accounted for $29(57 \%)$ cases of gravidity and $19(37 \%)$ cases of gravidity out of the total 51 cases studied. Figure 3 show the correlation between ethnicity and fibroids, polycystic ovaries and both for the four-year period in Trinidad. It can be seen from the Fig. 2 that Blacks have the highest rate of fibroids accounting for 207 (44\%) cases, followed by Indians with 189 (40\%) and lastly mixed with $76(16 \%)$ cases. Indians have a higher incidence of Polycystic Ovarian Syndrome: 22 (43\%) and for both conditions Blacks have the highest percentage $22(52 \%)$.

Figure 4 shows the relation between fibroids, polycystic ovarian syndrome and age in Trinidad during years 2000-2003. There were no cases registered for women under the age of 20 for fibroids while there were $8(16 \%)$ registered cases of polycystic ovarian syndrome, for women of 20-29 years there were 21 and 20 cases for fibroids and polycystic ovarian syndrome respectively. There were 122 cases of fibroids and 15 cases of polycystic ovarian syndrome for women between the ages of 30-39. There were 251 cases of fibroids and 7 cases of polycystic ovarian syndrome for women of ages between 40 and 49. There were 68 cases of fibroids and no cases of polycystic ovarian syndrome for women aged between 50 and 59; for women greater than 60 years there were 2 cases of fibroids and only 1 case of polycystic ovary.

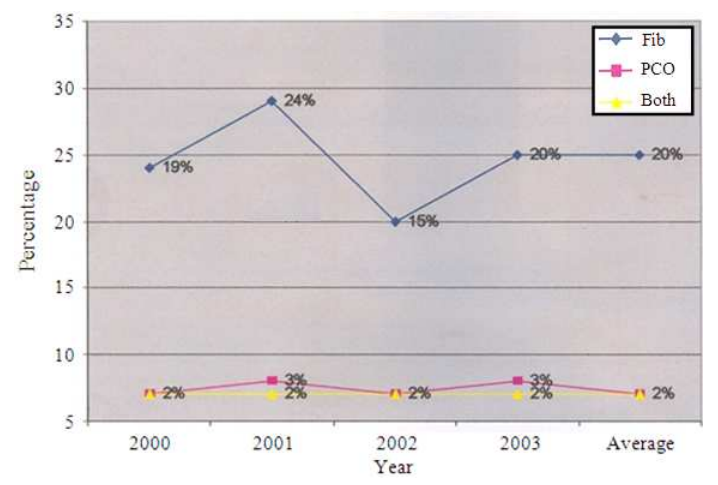

Fig. 1: Incidence of fibroids, polycystic ovarian syndrome and both in Trinidad (2000-2003)

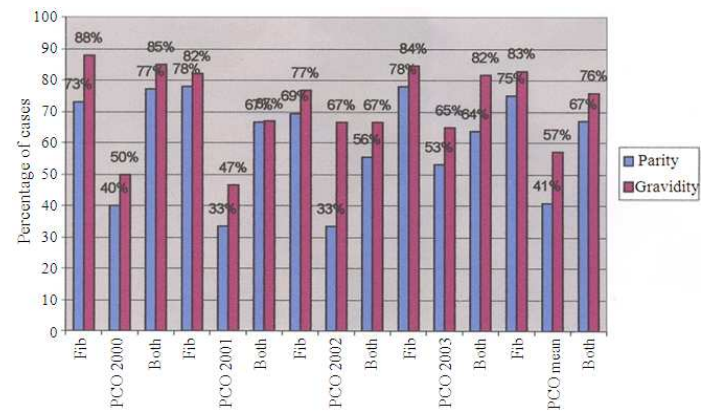

Fig. 2: Percentage parity and gravidity in Trinidadian women on with fibroids, polycystic ovaries and both (2000-2003) 
OnLine J. Biol. Sci., 9 (4): 86-92, 2009
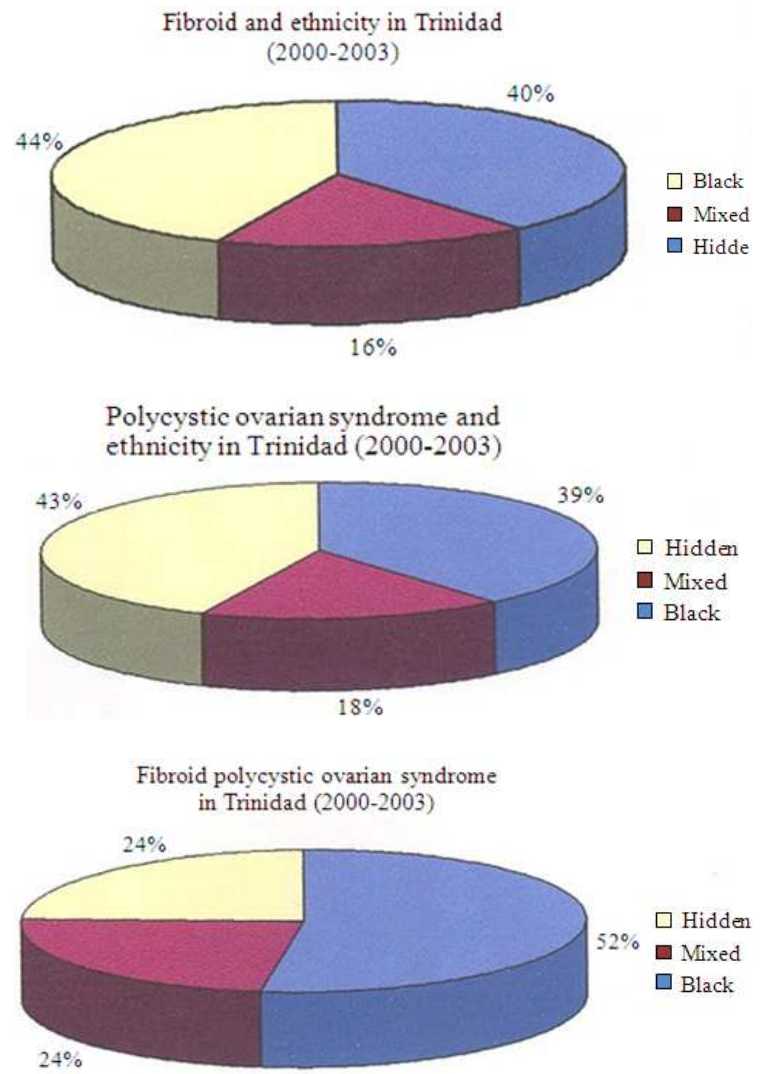

Fig. 3: Comparison between the ethnic groups and the different conditions in women in Trinidad (2000-2003)

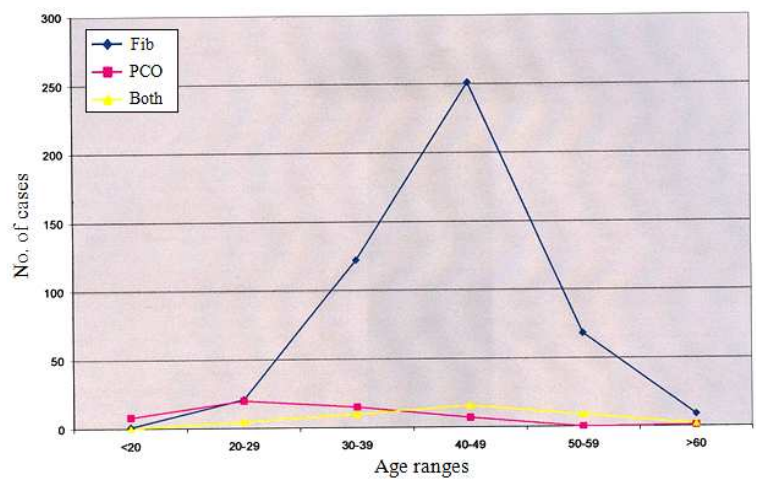

Fig. 4: Graph showing number of cases fibroids, PCO and both versus age range in Trinidad (20002003)

Figure 4 also shows that there were a greater number of cases for women in their late reproductive years for fibroids and more cases in early reproductive years for women with polycystic ovary syndrome.
Table 1: Summary table

\begin{tabular}{|c|c|c|c|}
\hline & Total No. of c & es (2400) & \\
\hline No. of cases & 473 & 51 & 42 \\
\hline & Fibroids (\%) & $\mathrm{PCO}(\%)$ & Both $(\%)$ \\
\hline Incidence & 20 & 2 & 2 \\
\hline Ethnicity & & & \\
\hline Black & 44 & 39 & 52 \\
\hline Mixed & 16 & 18 & 24 \\
\hline Indians & 40 & 43 & 24 \\
\hline Age ranges: & & & \\
\hline$<20$ & 1 & 16 & 0 \\
\hline $20-29$ & 4 & 39 & 12 \\
\hline $30-39$ & 26 & 29 & 24 \\
\hline $40-49$ & 53 & 14 & 38 \\
\hline $50-59$ & 14 & 0 & 21 \\
\hline$>60$ & 2 & 2 & 5 \\
\hline Parity & 75 & 41 & 67 \\
\hline Gravidity & 83 & 57 & 76 \\
\hline Presenting complai & & & \\
\hline Pain & 17 & 43 & 33 \\
\hline Menorrhagia & 25 & 6 & 12 \\
\hline Pelvic BI• & 26 & 27 & 31 \\
\hline Anemia & 9 & 10 & 5 \\
\hline Amenorrhea & 4 & 4 & 2 \\
\hline Other & 15 & 10 & 17 \\
\hline Treatment: & & & \\
\hline Hysterectomy & 70 & 6 & 67 \\
\hline Myomectomy & 10 & & 14 \\
\hline Ovarian cystectomy & 1 & 37 & 7 \\
\hline Hormone therapy & & & 25 \\
\hline Blood transfusion & 6 & 6 & \\
\hline Other/none & 12 & 43 & 5 \\
\hline
\end{tabular}

Table 1 displays the most common presenting complaint of persons with fibroids or polycystic ovarian syndrome and/or those with both. Throughout the 4 year period (2000-2003) 123 (26\%) presented with complaints of vaginal bleeding out of the four hundred and 73 fibroid cases. Another common occurrence in the fibroid cases was menorraghia, which was reported in 100 and $18(25 \%) 22$ patients (43\%) out of the 51 who presented with POC complained of pain, including pelvic and abdominal pain. This was the commonest complaint. The second most common complaint was vaginal bleeding. This accounted for 27\% (14 patients) of the total of fifty-one cases reported. In the case where both conditions viz. Leiomyomata and polycystic ovary were present, 14 patients (33\%) complained of pain and thirteen $(31 \%)$ complained of vaginal bleeding out of a total of 42 patients.

Table 1 also shows the most common types of treatment for fibroids and polycystic ovarian syndrome. Of the fibroid cases 331 (70\%) out of 473 cases had hysterectomy. Of the 51 polycystic ovarian cases, 22 (43\%) received some other forms of treatment while nineteen cases (37\%) had ovarian cystemectomies. Cases with both conditions were reported to have 28 
(67\%) receiving hysterectomy as the most common form of surgical intervention.

\section{DISCUSSION}

The results of the project showed that leiomyomata account for $473(20 \%)$ cases while polycystic ovaries accounted for 51 (2\%) of all the 2400 cases studied. Cases with both diseases accounted for 42 (2\%) cases. The results for the incidence of leiomyomata are similar to those obtained in other research that was previously conducted in the Barbados Fertility Center ${ }^{[10]}$, which found that leiomyomata are common, occurring in $20 \%$ of women studied, as we also obtained in the present study. Our incidence of polycystic ovary cases is slightly less than the value obtained in studies conducted in the United States of America by the University of Louisiana-they found the incidence to be around $6-10 \%$.

The present study shows that Blacks have the higher incidence of leiomyomata accounting for $44 \%$ of the 473 cases, followed by Indians which accounted for $40 \%$ and lastly by women of mixed races which accounted for only $16 \%$. The reason for this may not be unconnected to the fact that the population of Trinidad is predominated by Blacks and Indians. It is in light of the same reason that the study was conducted in the major hospitals in the north and south because of the distribution of the races between these two regions of the country.

The results of the present study are consistent with the contention of Hacking in 2004 that statistics shows that $25 \%$ of white women were likely to develop leiomyomata, $40-50 \%$ of East Indian women will be afflicted by the problem, while women of African origin are at a highest risk with about $75 \%$ developing the problem. The results we obtained also show that Blacks are at higher risk of having fibroids. The results are also consistent with those of the study reported in Merck Manual ${ }^{[11]}$, which states that fibroids are the most common pelvic neoplasm occurring in half the population of Blacks. Although the basis for the higher prevalence among black women is unknown, ethnic differences have been found in circulating estrogen levels while on control diets and differences in estrogen metabolism have also been noted. In control groups of healthy, premenopausal women placed on a high-tat, low-fiber diet similar to their usual diet, AfricanAmerican women had shown significantly higher serum levels of estrone, estradiol and free estradiol than Caucasian women ${ }^{[12]}$.

The present study also shows that Indians have more cases of polycystic ovary, accounting for $43 \%$, followed by Blacks with $39 \%$ and women of mixed races accounted for $18 \%$. There was no information obtained from previous studies that explained why women of Indian origin in Trinidad have a higher risk of polycystic ovary than women of other races however, previous studies have shown that there is a strong positive correlation between diabetes and obesity and polycystic ovary syndrome. One might at first glance assume that the data suggests that Indian women in Trinidad have more cases of obesity and diabetes, however, one explanation we propose for this is that the population of Indian women is greater in Southern Trinidad and thus this is reflected by the data we collected from San Fernando Hospital. No conclusion can be drawn on this subject without further studies.

Figure 4 shows that women aged between 40 and 49 have the highest cases of fibroids accounting for $251(53 \%)$ cases, followed by women aged between 30 and 39 with $122(26 \%)$. Women aged between 50-59 registered $68(14 \%)$ and women aged between 20-29 had $21(4 \%)$ cases. Only 9 cases $(3 \%)$ were registered for women greater than 60 years, while women less than 20 years did not register any case of fibroids. This data is similar to those of previous studies from the Barbados Fertility Center that have shown that fibroids are common in women aged between 30 and 50, during the later stages of their reproductive years ${ }^{[10]}$. An increase with age in the prevalence of fibroids during the reproductive years has also been demonstrated by several epidemiologic studies $^{[11]}$. A possible explanation for this scenario is that fibroids are oestrogen dependent. Consequently they enlarge in pregnancy and on the combined oestrogen-progesterone birth control pill and atrophy after menopause. They may degenerate gradually or suddenly (red degeneration). Occasionally they calcify ("womb stones"). Rarely, they become malignant, causing pain, malaise, bleeding and increase in size in postmenopausal women $^{[14]}$.

Our data also show that women aged between 20 and 29 have the highest cases of polycystic ovary, accounting for 20 (39\%) cases, followed by women aged between 30-39 who registered 32 (29\%) cases. Women less than 20 years registered 8 (16\%), while women aged between 40-49 years registered 7 (14\%). There were no cases recorded for women aged between 50-59, while only 1 (2\%) was registered for women greater than 60 years of age. These findings are similar to those of previous studies which indicated that polycystic ovary syndrome is a disorder of young women and is associated with patient exhibiting excessive production of androgens, increased conversion of androgens to estrogen and inappropriate 
gonadotropin production by the pituitary gland ${ }^{[4]}$. The increase in oestrogen is also the result of an abnormal pulsatile secretion of gonadotropin releasing hormone, which leads to increased amounts of Luteinizing Hormone (LH) and Follicular Stimulating Hormone (FSH) secretions. This subsequently results in hyperplasia of the ovarian theca cells leading to multiple follicular cysts and excessive androgen synthesis and anovulation.

As regards presenting complaints, previous studies have provided somewhat similar results.

A study done in Barbados Fertility Center in 2004 recorded up to half $(50 \%)$ of all the women with fibroids complained of having heavy pelvic bleeding or periods ${ }^{[10]}$. These results are consistent with the findings of the present study (Table 1). In 1998, a study on the cases of fibroids was carried out in Trinidad, but the total number of cases (130) used was too small for statistical analyses ${ }^{[15]}$. There were no conclusive statistics to solidify the actual percentage for the most common presenting complaint. However, the radiology consultant stated that the most common complaints of the fibroid cases were abdominal pain and heavy or prolonged pelvic bleeding ${ }^{[14]}$.

The study conducted at the Barbados Fertility Center on fibroid cases also recorded hysterectomies as their most common type of treatment used ${ }^{[10]}$. Based on the Barbados studies in conjunction with the data obtained in this study, it can be concluded that the most prevalent form of treatment used in the past few years have been hysterectomies, which entails the surgical removal of the uterus. The other, less frequently used, form of treatment was myomectomy, which entails the surgical removal of the individual fibroids. Considering the age group mostly affected by fibroids 40-49 years (Table 1), one can assume that having a hysterectomy would have no bearing on the patient's interests of childbearing since most women in this age group would have passed the age of childbearing and would probably be experiencing menopause. The data obtained from Fig. 2 shows that there is a high difference between gravidity and parity for women with polycystic ovary than for those with fibroids. The data also shows that there is high number of cases for parity and gravidity for women with fibroids than for women with polycystic ovary. The reason for this might be because polycystic ovary syndrome has an earlier onset during reproductive life and causes hyperplasia of the ovary and anovulation, while fibroids starts at a later stage of reproductive life while most women affected would already have had children.

An explanation that has sometimes been cited in the literature ${ }^{[16]}$ for these findings is that pregnancy reduces the time of exposure to unopposed estrogens, whereas nulliparity or reduced fertility may be associated with anovulatory cycles characterized by long-term unopposed estrogens. The alternative possibility exists that uterine fibroids are actually the cause of the infertility, rather than the consequence of it; however, the diminished relative risk of fibroids associated with parity remains essentially the same after exclusion of women with a history of infertility ${ }^{[13]}$.

The incidence of fibroids and polycystic ovary occurring together was very small. This might be because fibroids occur later in reproductive life while polycystic ovary occurs early in reproductive life. The other reason might be that fibroids are oestrogen dependent while polycystic ovary is androgen dependent.

\section{CONCLUSION}

The findings of the present study can be harnessed in improving the health of the population of Trinidad in different ways. For example, people can become aware of the different treatments of fibroids and most signs of fibroids and polycystic ovary and the most affected age group and ethnic group as well as other associated risk factors. The study can also be used as a template for future studies as it is to the best of our knowledge the only study to determine the incidence of fibroids and polycystic ovary in Trinidad.

\section{REFERENCES}

1. Goldmann, D.R. and D.A. Horowitz, 1999. American College of Physicians Complete Home Medical Guide. DK Publishing, New York, USA., ISBN: 10: 0789444127, pp: 1104.

2. Ballinger, A. and S. Patchett, 2003. Clinical Medicine. 3rd Edn., WB Saunders Company, pp: 555-556.

3. Johnson, M.E., 2002. Fibroids 101. Heart and Soul Mag., 1: 84-86.

4. Kumar, V., R. Cotran and S. Robbins, 2003. Basic Pathology. 7th Edn., WB Saunders Company Publishing, pp: 695.

5. Cool Nurse. Copyright 2000-2005. http://www.coolnurse.healthologycomlfocus_articl e. $8 \$ \mathrm{P} ? \mathrm{f}=$ women $\& \mathrm{~b}=$ coolnurse $\& \mathrm{c}=$ women_fibroids 03-02-05

6. Hirschberg, A.L., S. Naessen, M. Sbridsberg, B. Bystrom and J. Honet, 2004. Impaired CCK secretion and disturbed appetite regulation in women with polycystic ovary syndrome. Gynecol. Endocrinol., 19: 79-87.

http://www.ncbi.nlm.nih.gov/pubmed/15624269 
7. Kumar, V., R. Cotran and S. Robbins, 2003. Basic Pathology. 7th Edn,. WB Saunders Company Publishing, pp: 692-3.

8. Warshowsky, A., M.D. Oumano and E. Healing, 2002. Fibroids: A Doctor's Guide to a Natural Cure. Simon and Schuster, Australia, ISBN: 10: 0743418247, pp: 288.

9. The Jean Hailes Foundation 02-25-05. http://www.jeanhailes.org.au/issues/oincos.htm

10. barbadosivf.org, 2009. Barbados fertility centre. Fibroids. http://www.barbadosivf.org/fibroids.htm

11. Beers, M.H. and R. Berkow, 1999. The Merck Manual of Diagnosis and Therapy. 17th Edn., John Wiley and Sons, ISBN: 10: 0911910107, pp: 2833.

12. Gordon, P.F., A. Janet and D. Darlene, 2003. Environmental Health Perspective. Stony Brook. Volume 111, New York, USA.

13. Marshall, L.M., D. Spiegelman, J.E. Manson, M.B. Goldman, R.L. Barbieri and M.J. Stampfer, 1998. Risk of uterine leiomyomata among premenopausal women in relation to body size and cigarette smoking. Epidemiology, 9: 511-517. http://cat.inist.fr/?aModele $=$ afficheN\&cpsidt $=2411$ 349
14. Collier, J.A.B., I.M. Longmore and T.J. Hodgetts, 1994. Oxford Handbook of C1inica1 Specialist. 4th Edn., University of Oxford, Oxford, UK.

15. The Trinidad Express. Copyright, 1998 http://www.trinidadexpress.com/index.pl/articlewo manIDa2?id=23443560

16. Parazzini, F., E. Negri, C. La Vecchia, L. Chatenoud, E. Ricci and P. Guarnerio, 1996. Reproductive factors and risk of uterine fibroids. Epidemiology, 7: 440-442. http://www.jstor.org/stable/3702066 